\title{
A smart self-regenerative lithium ion supercapacitor with a real-time safety monitor
}

Xu-Yi Shan ${ }^{\mathrm{a}}$, Yuzuo Wang ${ }^{\mathrm{a}, \mathrm{b}}$, Da-Wei Wang ${ }^{\mathrm{c}}$, Zhe Weng $^{\mathrm{a}}$, Feng Li ${ }^{\mathrm{a}}{ }^{*}$ and Hui-Ming Cheng $^{\mathrm{a}}$

${ }^{a}$ Shenyang National Laboratory for Materials Science, Institute of Metal Research, Chinese Academy of Sciences, Shenyang, 110016, China.

${ }^{\mathrm{b}}$ Key Laboratory for Anisotropy and Texture of Materials, Northeastern University, Shenyang, 110819, China.

${ }^{c}$ School of Chemical Engineering, The University of New South Wales, Sydney, NSW 2052, Australia.

${ }^{*}$ Corresponding author:

Feng Li, Prof.

Shenyang National Laboratory for Materials Science

Institute of Metal Research, Chinese Academy of Sciences

72 Wenhua Road, Shenyang 110016, China

Tel: +86-24-8397 0065; Fax: +86-24-2390 3126

Email: fli@imr.ac.cn 
Abstract Lithium ion capacitors (LICs) show great promise for electricity storage. However, cycle degradation and unbalanced electrode kinetics of LICs are troublesome. Meanwhile, safety has become a stringent requirement for lithium devices. Finding methods to tackle these problems is rather challenging. Here, we develop a smart lithium ion supercapacitor (SLIC) by implanting a lithium electrode into a twoelectrode pouch cell. The proof-of-concept SLIC has three smart functions: (1) energy boosting: it supports an on-line electrochemical charge injection protocol that boosts the energy of symmetrical all-carbon supercapacitors. A SLIC with single-wall carbon nanotubes (SWCNTs) as both electrodes has a gravimetric energy 5 times higher than conventional supercapacitors using SWCNTs and excellent durability with no measurable degradation during 10,000 cycles; (2) safety monitoring: it allows real-time diagnosis of cell decay and warns of dangers by a built-in feedback mechanism; (3) self-regeneration: it enables regeneration of a degraded SLIC by a voltage modulator. From a practical point of view, this SLIC concept offers a general solution for extending the life of sealed power sources.

Keywords smart, supercapacitor, self-regenerative, safety monitor, energy boosting 


\section{Introduction}

To meet the ever-increasing needs of portable electronics, electric vehicles and gridscale renewable energy storage, a substantial increase in the energy and power densities of electrochemical energy storage devices (ESDs) has become urgent. ${ }^{[1-4]}$ However, as far as practical ESDs are concerned, neither lithium-ion batteries (LIBs) nor supercapacitors (SCs), can fulfill the projected energy and power requirements. ${ }^{[5-}$ 7] High energy LIBs make use of an intercalation-based reaction, but their slow diffusion kinetics leads to very low power. ${ }^{[3,8]}$ SCs have limited energy output despite their high power density. ${ }^{[2,9-13]}$

To bridge the gap between LIBs and SCs, research has focused on the development of hybrid ESDs. ${ }^{[14-16]}$ For example, a lithium ion capacitor (LIC) combines a graphite negative electrode with an activated carbon positive electrode where the mass of each electrode is different in order to match the capacity. ${ }^{[3,14-16]}$ Despite its high energy/power performance, the LIC has encountered drawbacks such as electrode degradation and unbalanced electrode kinetics. ${ }^{[17]}$ Recently, our group has developed an electrochemical charge injection (ECI) protocol to adjust the electrode kinetics and optimize the capacity of LICs with symmetric carbon electrodes. By modulating the initial state of charge, the ECI protocol can balance the charge accumulation rate on each electrode and maximize the cell voltage to the upper limit of the stable electrochemical window of the lithium ion electrolyte used. Compared to conventional LICs, this ECI-LIC system used the same amount of active materials in both the positive and negative electrodes, and showed superior energy density and power density which is promising for industrial applications. ${ }^{[18]}$ Unfortunately, a tedious prelithiation step by disassembly and reassembly, i.e. off-line ECI, is required. A substantial design of device structure has to be implemented.

Furthermore, in the use of lithium-based ESDs, safety is the most stringent requirement. ${ }^{[5,7,19]}$ The many reported incidents of device explosion are usually related to lithium metal plating, dendrite formation and electrolyte decomposition. 
These hazardous electrochemical processes occur when electrode potentials are out of the safety range. It is essential to have real-time monitoring of the electrode potential in order to predict and prevent accidents. Furthermore, a LIC suffers from performance decay which is also caused by the shift of the electrode potentials. ${ }^{[20]}$ It is possible that by restoring the electrode potentials to a safe state, the performance of LICs can be reactivated.

Herein, we report a smart lithium ion supercapacitor (SLIC) that has a built-in safety monitor to mitigate risks and to regenerate its capacity if the cell loses $75 \%$ of its initial capacity. The SLIC was built by simply introducing a lithium electrode as a voltage modulator, in addition to the positive and negative electrodes, to monitor and adjust potential fluctuations on both the positive and negative electrodes during cycling, and to fulfill on-line ECI to boost energy density. This built-in voltage modulator allows a real-time diagnosis of capacity decay, safety control through a built-in feedback system, and the regeneration of a degraded SLIC.

\section{Experimental}

Synthesis of carbon materials. The single-wall carbon nanotubes (SWCNTs) used were prepared by chemical vapor deposition, where methane was decomposed over an $\mathrm{Fe} / \mathrm{Mo} / \mathrm{MgO}$ catalyst, and purification was then carried out to remove residual metal. ${ }^{[21-23]}$ The preparation of chemically derived graphene $(G)$ was described in detail in our previous work. ${ }^{[24]}$ The hierarchical porous graphitic carbon (HPGC) was prepared by carbonizing phenolic resin with $\mathrm{Ni}(\mathrm{OH})_{2}$ as a template. ${ }^{[25]}$ The commercial SWCNTs (com-SWCNTs) $(<2 \mathrm{~nm}$ diameter, 5 15 $\mu \mathrm{m}$ length) and com-MWCNTs $(<10 \mathrm{~nm}$ diameter, 5 15 $\mu \mathrm{m}$ length) were obtained from Shenzhen Nanotech Port Co, Ltd, China. The commercial activated carbon (AC) (YP-50F) (about $6 \mu \mathrm{m}$ diameter and BET surface area about $1600 \mathrm{~m}^{2} / \mathrm{g}$ ) was obtained from Kuraray Chemical Co., Ltd.

Materials Characterization. The nano-carbon materials were characterized by SEM (FEI Nova NanoSEM-430) and TEM (FEI Tecnai F20, $200 \mathrm{kV}$ ), the specific surface area and pore size distribution were measured by nitrogen adsorption/desorption on a 
Micromeritics ASAP 2020 instrument, XPS measurements were performed using an ESCALAB250 with A1 K $\alpha$ radiation, and Raman spectra were collected with a JY HR800.

Fabrication of SLICs. Electrodes were prepared by mixing active materials with poly(tetrafluoroethylene) (PTFE) and acetylene black (Super-P), and then pressed on stainless steel 304 mesh. The mass ratios of active material, PTFE and Super-P were 80:10:10 for G and HPGC, due to their low tap density, and 90:5:5 for SWCNTs, comSWCNTs, com-MWCNTs and AC (YP-50F). The mass loading for all the electrodes was $5 \mathrm{mg}$. To fabricate SLIC cells, a solution of $1 \mathrm{~mol} \mathrm{~L}^{-1}$ lithium hexafluorophosphate $\left(\mathrm{LiPF}_{6}\right)$ in a mixture of ethylene carbonate (EC) and diethyl carbonate (DEC) ( $\mathrm{vol} / \mathrm{vol}=1: 1)$ served as the electrolyte and a polyethylene separator was used as the separator. First, aluminum and nickel tabs were respectively welded to positive and negative electrodes. Then, a piece of lithium foil $(60 \mu \mathrm{m}$ in thickness) was pressed on a nickel tab serving as the $3^{\text {rd }}$ electrode. The cell core was obtained by placing electrodes in the order of positive electrode (PE)/ negative electrode (NE)/ lithium electrode (Li), separated by polyethylene separators. For Stack-Two and Stack-Four SLIC devices, the corresponding PE and NE were alternately stacked, separated by polyethylene separator, and then a lithium electrode was placed on the top. The device cores were packaged with aluminium-laminated films by an edge bonding machine. Finally, the injection of the electrolyte was conducted in an argon-filled glove box followed by sealing.

Determination of $\boldsymbol{E}^{\prime}{ }_{\boldsymbol{o v}}$. Firstly, the PE and NE working electrodes were galvanostatically charged/discharged relative to the lithium voltage modulator/electrode at $0.875 \mathrm{~A} / \mathrm{g}$ between $4.31 \mathrm{~V}$ and $0.01 \mathrm{~V} v s . \mathrm{Li} / \mathrm{Li}^{+}$for 20 cycles to achieve a stable state by using the lithium electrode as the counter electrode, separately. The intersection point between the discharge plot for NE and the charge plot for PE was taken as the $E^{\prime}{ }_{o v}$. Ideally, when the initial potential of both PE and NE was $E^{\prime}{ }_{o v}$, the potential of $\mathrm{PE}$ and $\mathrm{NE}$ will reach $4.31 \mathrm{~V}$ and $0.01 \mathrm{~V}$ simultaneously to achieve a $4.3 \mathrm{~V}$ high voltage after full charge of the device. 
On-line ECI. A 4.3V was chosen as the cell voltage in accordance with our previous work. ${ }^{[18]}$ After the determination of $E^{\prime}{ }_{o v}$, on-line ECI was conducted by holding the PE and NE electrodes at $E^{\prime}{ }_{o v}$ relative to lithium electrode for $2 \mathrm{~h}$ to reach an equilibrium simultaneously inside the pouch cell. After the preliminary treatment, upon a full charge, a SLIC with on-line ECI with a predetermined cell voltage of $4.3 \mathrm{~V}$ was obtained.

Electrochemical Measurements. The galvanostatic charge/discharge tests were done by using a VSP-300 multichannel potentiostat/galvanostat at room temperature. The energy density (E) and the power density (P) of SLIC devices were calculated from the following equations: ${ }^{[26]}$

$\begin{array}{lllllll}\mathrm{P} & = & \mathrm{V} & \mathrm{i} & / & 2 \mathrm{~m}\end{array}$

$\begin{array}{lllllll}\mathrm{E} & = & \mathrm{P} & \mathrm{x} & \mathrm{t} & / & 3600\end{array}$

$\Delta \mathrm{V} \quad=\quad \mathrm{E}_{\max } \quad-\mathrm{E}_{\min }$

$E_{\max }$ and $E_{\min }$ are the voltages at the beginning and end of the discharge $(V), i$ is the discharge current (A), $\mathrm{t}$ is the discharge time (sec.), and $\mathrm{m}$ is the total mass of active materials in the anode and cathode $(\mathrm{g})$.

\section{Results and discussion}

A schematic of the SLIC is given in Figs. 1a and b. Different from a commercial SC pouch cell, the SLIC has three electrodes: a positive electrode $(\mathrm{PE})(+)$, a negative electrode (NE) (-) and a lithium electrode as a voltage modulator (Li) (Figure 1a). Specifically, two identical positive and negative electrodes made of nano-carbon materials and lithium are stacked in the order $\mathrm{PE} / \mathrm{NE} / \mathrm{Li}$, and these are separated by polyethylene separators (Figure 1b). After injecting the electrolyte, a SLIC is obtained. Details of the fabrication are given in the Experimental section. Figure 1c shows a photograph of the assembled SLIC. The lithium voltage modulator has four specific 
functions here: (1) as a reference electrode to measure voltage fluctuation ${ }^{[18,20]} ;(2)$ as an alarm if the electrode potential exceeds the safety limit; (3) as a sensor to determine the degree of cell degradation; and (4) as a built-in lithium source for an on-line ECI protocol to revive the degraded cells.

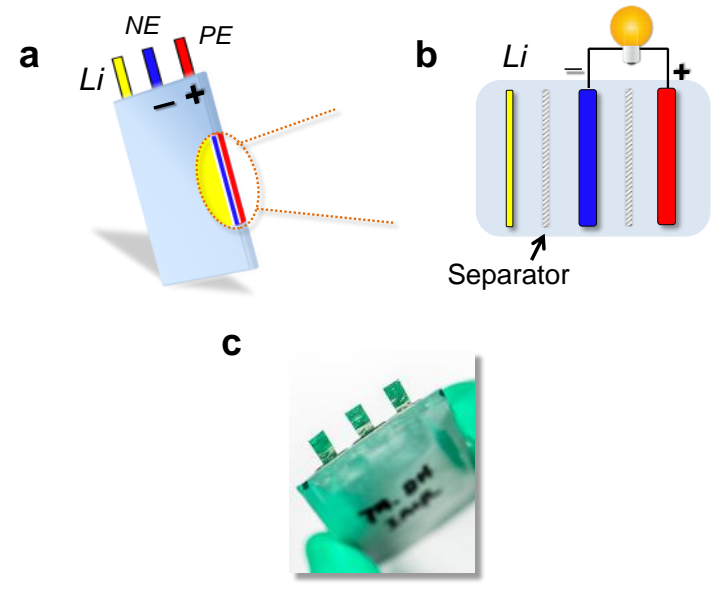

Figure 1 Illustration of a SLIC. a. A SLIC with three electrodes. The three electrodes are PE (+), Li, NE (-). b. Internal construction of a SLIC. c. Photograph of an assembled SLIC.

A maximum cell voltage of $4.3 \mathrm{~V}$ which was close to the stable voltage window of the electrolyte was chosen in accordance with our previous work. ${ }^{[18]}$ Compared with the previous off-line ECI method through disassembly and reassembly, we developed a simple on-line protocol for practical use. In this situation, the whole ECI was finished inside the pouch cell. The protocol was schematic shown in Figure 2a. Firstly, the PE and NE working electrodes were galvanostatically charged/discharged relative to the lithium voltage modulator/electrode at $0.875 \mathrm{~A} / \mathrm{g}$ between $4.31 \mathrm{~V}$ and $0.01 \mathrm{~V} v$ s. $\mathrm{Li} / \mathrm{Li}^{+}$for 20 cycles to achieve a stable state by using the lithium electrode as the counter electrode, separately. The intersection between the discharge plot for $\mathrm{NE}$ and the charge plot for PE was taken as the $E^{\prime}{ }_{o v}$. Secondly, On-line ECI was conducted by holding the PE and NE electrodes at $E^{\prime}{ }_{o v}$ relative to lithium electrode for $2 \mathrm{~h}$ to reach an equilibrium simultaneously inside the pouch cell. After these preliminary treatments, upon a full charge, a SLIC with on-line ECI with a 
predetermined cell voltage of $4.3 \mathrm{~V}$ would be obtained. To verify the on-line protocol, we selected single-walled carbon nanotubes (SWCNTs) with few defects as the electrode material. ${ }^{[21]}$ The potential of the SWCNT electrodes at the intersection point between the charge profile of PE (blue) and the discharge profile of NE (red) is denoted $E_{o v}$ (the point of equal capacity for PE and NE) (Figure 2b). Then, on-line ECI was conducted by holding the two SWCNT electrodes at $E^{\prime}{ }_{o v}$ for $2 \mathrm{~h}$ to reach equilibrium. Upon full charge, a SLIC with $4.3 \mathrm{~V}$ was obtained (red), $1.5 \mathrm{~V}$ higher than a conventional SWCNTs-SC (2.8V) (blue) (Figure 2c). Compared with the SWCNTs$\mathrm{SC}$, the discharge capacity of the SWCNTs-SLIC was much higher at discharge current densities from $0.875 \mathrm{~A} / \mathrm{g}$ to $5.25 \mathrm{~A} / \mathrm{g}$ (Figure $2 \mathrm{~d}$ ). According to the formula $\mathrm{E}=1 / 2 \mathrm{QU}$, where $\mathrm{Q}$ is the specific capacity and $\mathrm{U}$ is the working voltage ${ }^{[27]}$, a simultaneous increase of $\mathrm{Q}$ and $\mathrm{U}$, produces a much higher energy density of the SWCNTs-SLIC ( $85 \mathrm{Wh} \mathrm{kg}^{-1}$ electrode) than that of a conventional one (16 $\mathrm{Wh} \mathrm{kg}^{-1}$ electrode), as shown in the Ragone plots (Figure 2e). The long-term cycling stability of SWCNTs-SLIC was tested by galvanostatic charge/discharge and no measurable degradation was observed even after 10,000 cycles. The gradual increase of capacity retention during the initial 5000 cycles was ascribed to the continuous penetration of electrolyte and activation of SWCNTs along with cycles. A coulombic efficiency greater than 99\% was achieved during cycling with $100 \%$ discharge (Figure 2f). The voltage profiles of the last three cycles (Inset of Figure 2f) were symmetric and near-linear with a small ohmic drop, implying good stability. By introducing a built-in lithium metal voltage modulator, we have easily implemented the ECI strategy into a practical device capable of on-line ECI operation. 
a

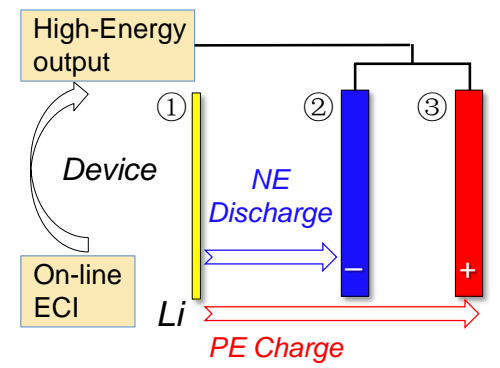

C

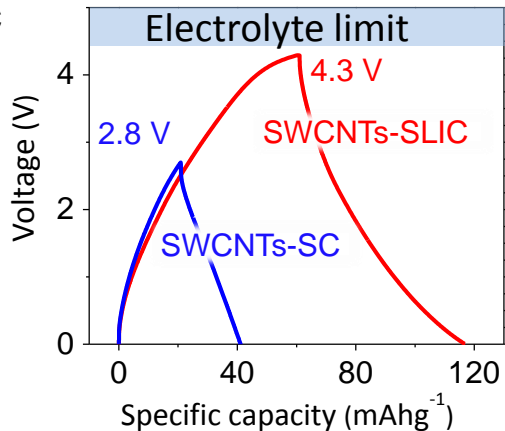

b

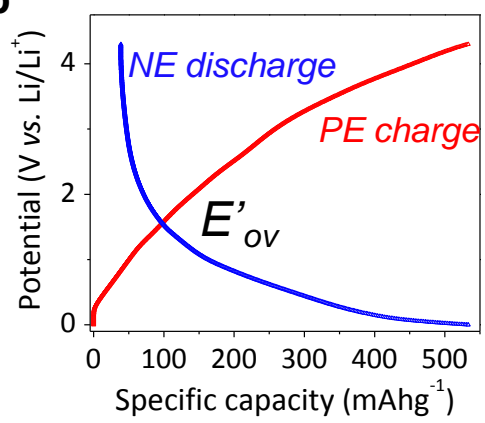

d

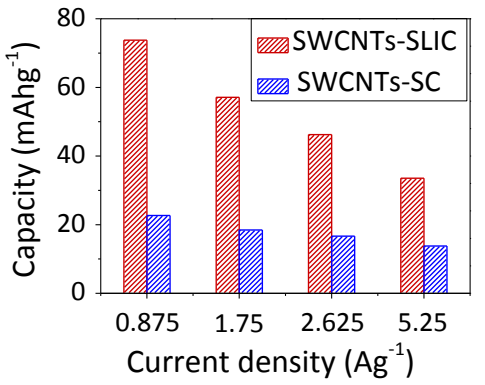

e



f

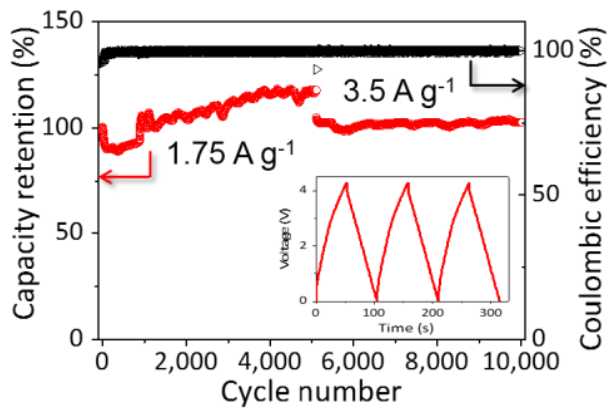

Figure 2 Electrochemical performance of SWCNTs-SLICs. a. Schematic of the online ECI protocol. b. Galvanostatic discharge profile of NE and charge profile of PE between $0.01-4.31 \mathrm{~V}$ at $0.875 \mathrm{~A} / \mathrm{g}$. The intersection is $\mathrm{E}_{\text {ov. }}$. c. Charge/discharge profiles of a SWCNTs-SLIC (red) and SWCNTs-SC (blue) at $1.75 \mathrm{~A} / \mathrm{g}$. d. Comparison of the rate performance of SWCNTs-SLIC (red) and SWCNTs-SC (blue) at different current densities. The calculation of specific discharge capacity was based on the active material per electrode. e. Ragone plots of SWCNTs-SLIC and SWCNTs-SC (based on the total mass of the active materials in positive and negative electrodes). The performance of conventional SCs, LIBs and $\mathrm{Ni} / \mathrm{MH}$ batteries was estimated from the values in the Ragone plots in a previous paper ${ }^{[1]}$ by multiplying by a factor of 3 , because the weight of the active material usually accounts for about $30 \%$ of the total mass of the packaged devices. ${ }^{[28]}$ f. Cycling stability and coulombic efficiency of a 
SWCNTs-SLIC during 10000 cycles. $1.75 \mathrm{~A} / \mathrm{g}$ for the initial 5000 cycles and $3.5 \mathrm{~A} / \mathrm{g}$ for the following 5000 cycles. Inset: The voltage profiles of the final three cycles.

Further, laminated SLIC devices were fabricated by stacking alternating positive and negative electrodes with a Li metal voltage modulator as a top layer (Figure 3). A Stack-X device denotes a SLIC with X-layers of positive and negative electrodes alternatively and a Li metal voltage modulator. The discharge time of a Stack-Four device was almost four times that of a Stack-One device at the same output current showing a linear relationship between capacity and the number of layers. (Figure 3a) The Stack-X devices showed good scalability to provide a high energy supply. Cycling stability is another important concern for LICs. A Stack-Two device showed little degradation in the specific capacity and a coulombic efficiency near $100 \%$ after 2000 cycles (Figure 3b), indicating good electrochemical stability. For a Stack-Four device, regardless of a rapid drop during the first 50 cycles, the capacity retention was stable at about $80 \%$ during the following 950 cycles (Figure 3c). The initial decay may be due to the manual stacking of the layers which was not accurate and can be improved. To further demonstrate the potential use of the Stack-Four device, a red light-emitting diode (LED) display of the IMR logo was illuminated (Figure 3d). Although the introduction of $3^{\text {rd }}$ lithium electrode will reduce the whole energy density of StackOne device, however, predictably, along with the increase of $\mathrm{X}$ in a device, the influence of Li electrode on the energy density should be negligible. 

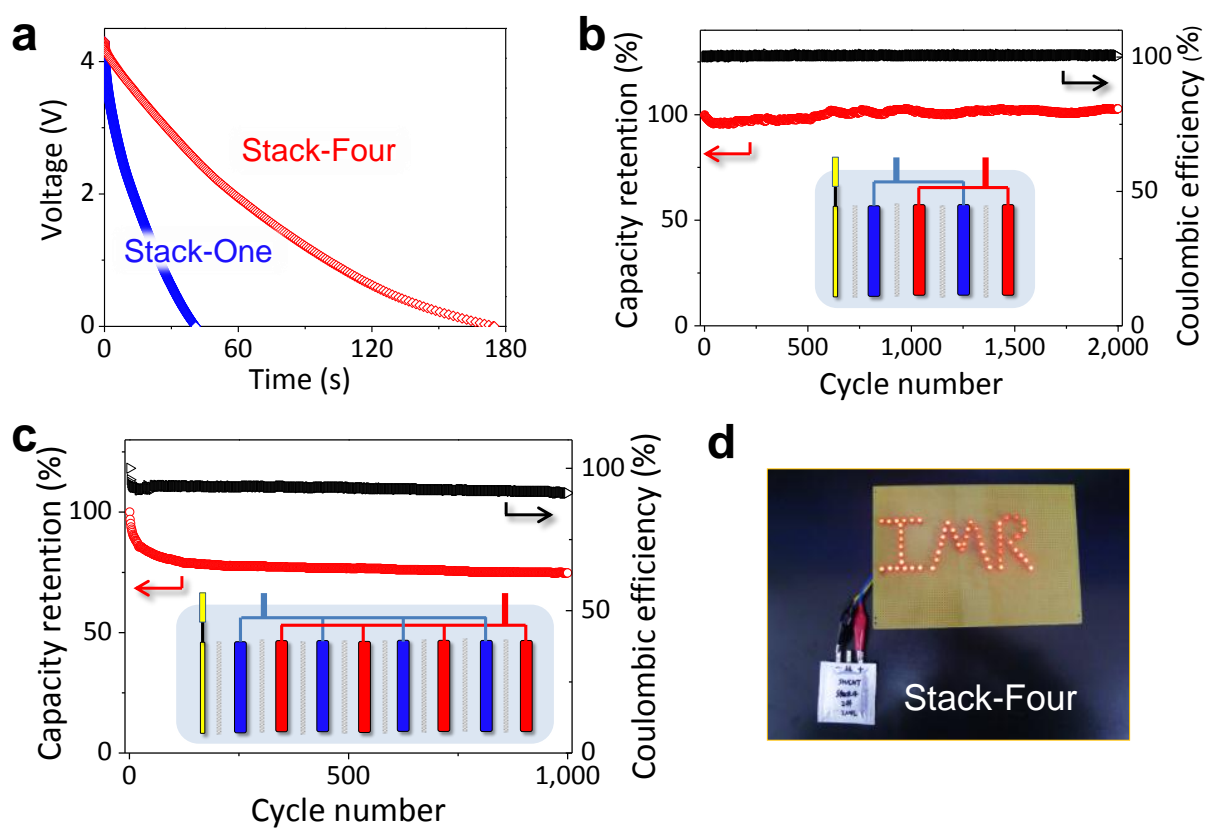

d

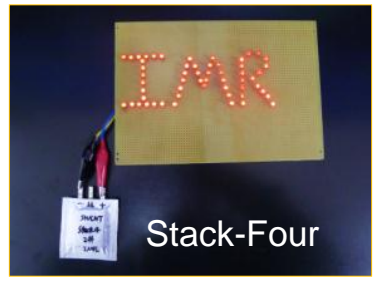

Figure 3 Electrochemical performance of stacked SWCNTs-SLICs. a. Galvanostatic discharge profiles of Stack-One and Stack-Four devices. b. Cycling stability and coulombic efficiency of a Stack-Two device. Inset: Schematic of internal construction. c. Cycling stability and coulombic efficiency of a Stack-Four device. Inset: Schematic of internal construction. d. Optical image of a red LED logo lit by a Stack-Four device.

To further investigate the stability of the SWCNTs in the electrodes after cycling, we extracted samples from the electrodes after 5000 cycles for observation with SEM and TEM (Figure S1). Even after 5000 cycles, the SWCNTs in the positive electrode were almost identical to the original ones, while for those from the negative electrode, their morphology was well retained but a thin solid electrolyte interface (SEI) layer was formed on their surface. To better understand the stability of SWCNTs in SLICs, we conducted nitrogen adsorption/desorption, X-ray photoelectron spectroscopy (XPS) and Raman spectroscopy characterization. The large Brunauer-Emmett-Teller (BET) specific surface area of $1260 \mathrm{~m}^{2} / \mathrm{g}$ and one-dimensional microstructure of SWCNTs ensures a high double-layer capacitance and short ion diffusion paths for high energy and power density (Figure S2). Meanwhile, an ultra-low Raman D/G intensity ratio (0.17) and low oxygen content (2.21 at\%) of SWCNTs (Table S1) indicated a nearperfect carbon structure with a high crystallization. The side reaction of SWCNTs with 
electrolyte during cycles would be minimized. Furthermore, for micropore-dominated SWCNTs determined by density functional theory (DFT) pore-size distribution (Inset of Figure S2), the ions and solvent molecules adsorbed may not be susceptible to side reactions because of the pore confinement. ${ }^{[29]}$ The electrodes are therefore stable and have a long cycle life.

Although SWCNTs exhibited extraordinary stability in SLICs, it is arguable that other nano-carbon materials would also be stable. We studied several nano-carbon materials that are more defective than SWCNTs, such as chemically derived graphene $(\mathrm{G})^{[24]}$, hierarchical porous graphitic carbon (HPGC) ${ }^{[25,30]}$ and activated carbon (AC), as well as commercial SWCNTs and multi-wall carbon nanotubes (MWCNTs) obtained from Shenzhen Nanotech Port Co., respectively designated com-SWCNTs and comMWCNTs (Table S1 and Figure S3). After an on-line ECI, the work voltage of SLICs based on these carbon electrodes all can reach $4.3 \mathrm{~V}$, which is consistent with SWCNTsSLIC (Figure S4) indicating the universality of the protocol. However, compared with the aforementioned SWCNT sample with an ultra-low Raman D/G intensity ratio $(0.17)$ and low oxygen content (2.21 at\%), these more defective and/or oxidized samples would have stronger side reactions with the electrolyte under $100 \%$ charge/discharge during cycling. As a result, the corresponding SLICs degraded at different levels during cycling (Figure S4f).

Safe operation of LICs is very important for applications in transportation, largescale renewable energy storage and next-generation portable electronics. ${ }^{[5,7,19]}$ It is known that the potentials of the positive and negative electrodes of conventional LICs using activated carbon and graphite shift from their initial values when cycled. ${ }^{[17,31-}$ ${ }^{33]}$ At the same time, the graphite anode has the risk of lithium plating at high currents due to unbalanced electrode kinetics. ${ }^{[27]}$ When the potential of the positive electrode increases to the limit for electrolyte decomposition $\left(4.5 \mathrm{~V} \mathrm{vs} \mathrm{Li} / \mathrm{Li}^{+}\right)$or the potential of the negative electrode goes below the potential of lithium plating ( $0 \mathrm{~V}$ vs $\left.\mathrm{Li} / \mathrm{Li}^{+}\right)$, safety issues such as swelling or lithium dendrite formation may occur. Therefore, we have 
designed a smart component to determine and provide an alert of the safety level of SLICs. As a consequence, the risk of lithium metal plating and electrolyte decomposition can be monitored and controlled. A schematic of the smart component (a feedback system) is shown in Figure 4a. It contains two voltage sensors $\left(\mathrm{V}_{1}\right.$ and $\left.\mathrm{V}_{2}\right)$ to monitor simultaneously the potential change of each electrode versus the lithium voltage modulator. During cycling, when $\mathrm{V}_{2}>4.5 \mathrm{~V}$ (exceeding the electrolyte decomposition limit) or $\mathrm{V}_{1}<0 \mathrm{~V}$ (lithium plating), the sensors send an alert and the device is turned off. This smart function effectively prevents swelling and Li dendrite formation, which may cause fire and explosion if uncontrolled. Using a graphenebased SLIC (G-SLIC) as an example, due to a continuous side reaction with electrolyte, the device underwent gradual degradation during cycling (red profile in Figure 4b), along with shifts of the maximum potential for PE ( $\mathrm{E}_{\mathrm{p}-\mathrm{max}}$, orange profile in Figure $4 \mathrm{~b}$ ) and the minimum potential for $\mathrm{NE}\left(\mathrm{E}_{\mathrm{n}-\mathrm{min}}\right.$, green profile in Figure $4 \mathrm{~b}$ ). Once $\mathrm{V}_{2}$ became higher than $4.5 \mathrm{~V}$ after 280 cycles, the G-SLIC was turned off (Figure 4b) to avoid intense side reactions. Predictably, the safety of conventional asymmetric LICs can be ensured by a similar safety monitor as well through this design. 

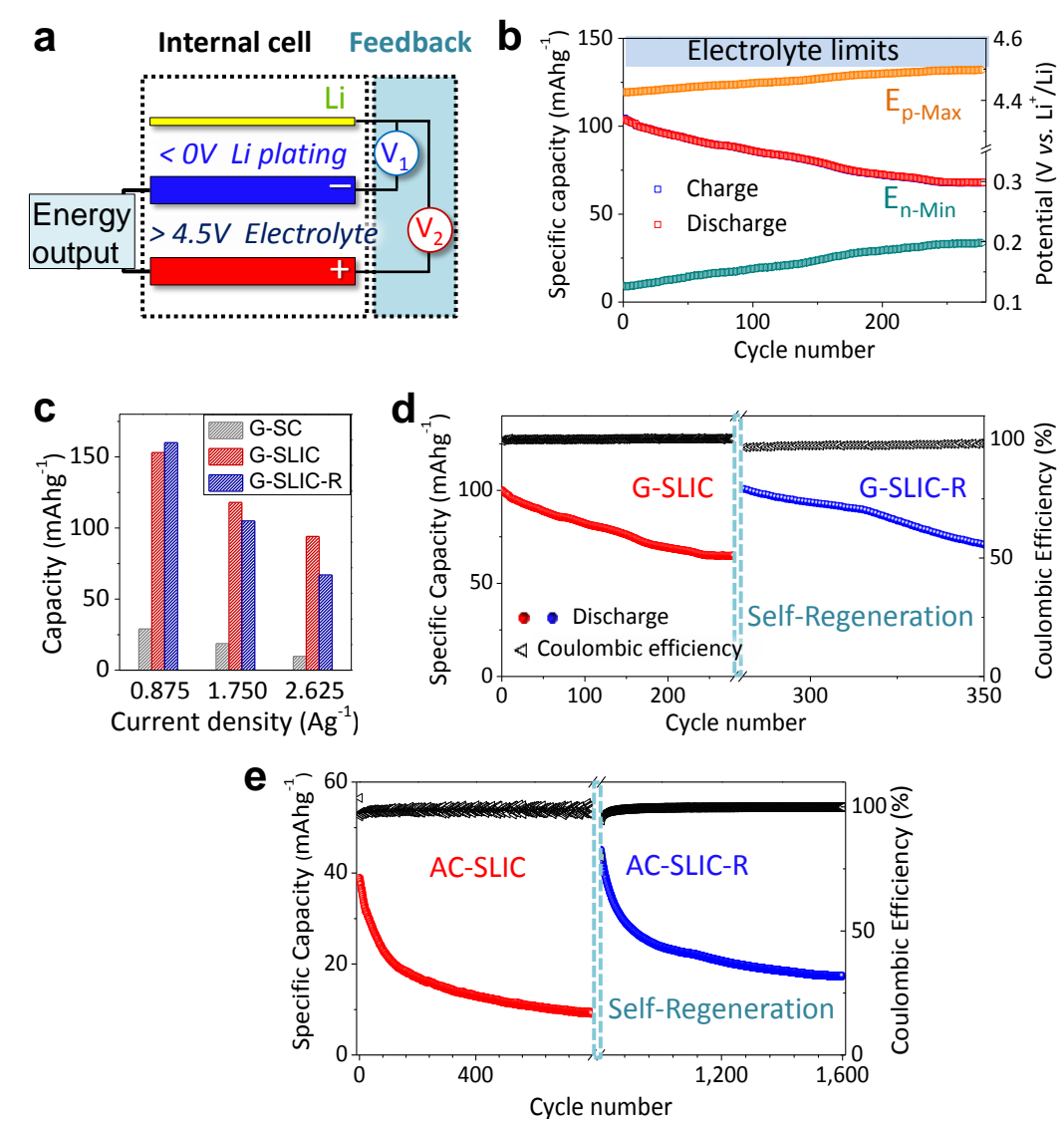

Figure 4 Experimental demonstration of the smart functions of SLICs. a. Schematic of the feedback system. b. Safety-monitoring for G-SLIC. c. Comparison of rate performance between a G-SLIC and a G-SLIC-R. d. The cycling stability of a G-SLIC and a G-SLIC-R. e. The cycling stability of an AC-SLIC and an AC-SLIC-R.

For practical uses, particularly for portable electronic products with sealed-in ESDs, the calendar-life of ESDs determines the service life of these electronics. To tackle the performance degradation of ESDs, much work has been done to improve electrode materials and electrolyte, in addition to developing new devices. This new SLIC has another smart function of self-regeneration to revive SLICs during service. As an example, we chose a degraded G-SLIC turned off by the feedback system. An on-line ECI treatment was conducted to refresh it, as shown in Figure 2a. We call this process self-regeneration. After self-regeneration the G-SLIC (G-SLIC-R) (blue in Figure 4c) exhibited rate performance approximate to the pristine G-SLIC (red in Figure 4c). Although the specific capacity of the G-SLIC decreased about $40 \%$ after 
280 cycles at $1.75 \mathrm{~A} / \mathrm{g}$ (red in Figure 4d), it was recovered to $105.3 \mathrm{~mA} \mathrm{~h} / \mathrm{g}$ after selfregeneration with a coulombic efficiency of about 100\% (blue line in Figure 4d). Due to the better wettability and higher accessible surface area developed during cycling, the capacity after regeneration was slightly higher than the initial value $(103.7 \mathrm{~mA} \mathrm{~h} / \mathrm{g})$. To further verify the universality of this smart function, we evaluated an AC-SLIC after degradation. After being turned off by its feedback system (Figure 4a) at 800 cycles, the AC-SLIC has undergone a 75\% capacity loss, but was regenerated to work well again (Figure 4e). Instead of disposal, self-regeneration offers a new perspective in dealing with degraded cells, which is obviously environment-friendly. The built-in feedback system can be readily interfaced with a battery management system (BMS), adding little cost to the whole supply system.

\section{Conclusion}

In summary, we have demonstrated a conceptual smart LIC containing a Li voltage modulator in addition to the positive and negative electrodes. The advantages of this $\mathrm{Li}$ voltage modulator, are that the SLIC has three unique functions. (1) Safetymonitoring: the potential change of the electrodes in a SLIC can be monitored in realtime. The voltage sensors of a simple feedback system provide an alert when the electrode potential exceeds the safe limit of the electrolyte, effectively preventing swelling and Li plating and improving safety. (2) Self-regeneration: it can on-line revive degraded devices to the initial state on-line for recycling use instead of disposal. By self-regeneration, a degraded G-SLIC and an AC-SLIC were revived, showing a great potential for practical applications, such as a sealed power supply in aerospace and other extreme environments which are unsuitable for power replacement. (3) Energy boosting: the ECI can be easily fulfilled on SLICs with various nano-carbons as electrode materials to increase their energy density. By using SWCNTs with few defects and a high specific surface area as the active material, a SWCNT-SLIC shows a high energy density ( $85 \mathrm{Wh} \mathrm{kg}^{-1}$ electrode at $1 \mathrm{~kW} \mathrm{~kg}^{-1}$ electrode) and impressive stability 
with no measurable degradation after 10,000 cycles. Laminated SLICs performed well suggesting a way to further increase the energy density. We believe that by using this concept with a programmed control-circuit, a high-energy, high-power and long-life SLIC can be developed. This smart device configuration should open up new opportunities for other ESDs such as conventional LICs and LIBs.

\section{Acknowledgement}

This work is supported by Ministry of Science and Technology of China (No. 2011CB932604 and 2014CB932402), National Science Foundation of China (Nos. 51221264, 51172239, 51372253 and U1401243), "Strategic Priority Research Program" of the Chinese Academy of Sciences (XDA01020304) and the Key Research Program of Chinese Academy of Sciences (Grant No. KGZD-EW-T06). The authors also thank the valuable discussion with Prof. Yang Yusheng, Dr. Zhang Qiang, Prof. Fei Wei and Mr. He Chiyuan.

\section{References}

[1] P. Simon, Y. Gogotsi, Nature materials 7 (2008) 845-854.

[2] J. Yan, Q. Wang, T. Wei, Z. Fan, Advanced Energy Materials 4 (2014) 1300816.

[3] D. P. Dubal, O. Ayyad, V. Ruiz, P. Gomez-Romero, Chemical Society Reviews 44 (2015) 1777-1790.

[4] D. Larcher, J.M. Tarascon, Nat Chemistry 7 (2015) 19-29.

[5] P. G. Bruce, B. Scrosati, J. M. Tarascon, Angewandte Chemie International Edition 47 (2008) 2930-2946.

[6] N. S. Choi, Z. Chen, S. A. Freunberger, X. Ji, Y. K. Sun, K. Amine, G. Yushin, L. F. Nazar, J. Cho, P. G. Bruce, Angewandte Chemie 51 (2012) 9994-10024.

[7] J. M. Tarascon, M. Armand, Nature 414 (2001) 359-367.

[8] H. D. Yoo, E. Markevich, G. Salitra, D. Sharon, D. Aurbach, Materials Today 17 (2014) 110-121. 
[9] F. Beguin, V. Presser, A. Balducci, E. Frackowiak, Advanced Materials 26 (2014) 2219-225.

[10]C. Liu, F. Li, L. P. Ma, H. M. Cheng, Advanced Materials 22 (2010) E28-E62.

[11]A. Ghosh, Y. H. Lee, ChemSusChem 5 (2012) 480-499.

[12]S. Zhang, N. Pan, Advanced Energy Materials 5 (2015) 1401401.

[13]Z. S. Wu, X. Feng, H. M. Cheng, National Science Review 1 (2013) 277-292.

[14]Q. Lu, J. G. Chen, J. Q. Xiao, Angewandte Chemie International Edition 52 (2013) 1882-1889.

[15]V. Aravindan, J. Gnanaraj, Y.-S. Lee and S. Madhavi, Chemical Reviews 114 (2014) 11619-11635.

[16]K. Naoi, Fuel Cells 10 (2010) 825-833.

[17]H. Kim, K. Y. Park, J. Hong, K. Kang, Scientific Reports 4 (2014) 5278.

[18]Z. Weng, F. Li, D. W. Wang, L. Wen, H. M. Cheng, Angewandte Chemie International Edition 52 (2013) 3722-3725.

[19]J. B. Goodenough, Y. Kim, Chemistry of Materials 22 (2010) 587-603.

[20]M. Schroeder, M. Winter, S. Passerini, A. Balducci, Journal of The Electrochemical Society 159 (2012) A1240-A1245.

[21]Q. Zhang, J. Q. Huang, M. Q. Zhao, W. Z. Qian, F. Wei, ChemSusChem 4 (2011) 864-889.

[22] Q. Zhang, J. Q. Huang, W. Z. Qian, Y. Y. Zhang, F. Wei, Small 9 (2013) 1237 1265.

[23] M. Q. Zhao, X. F. Liu, Q. Zhang, G. L. Tian, J. Q. Huang, W. C. Zhu, F. Wei, ACS Nano 6 (2012) 10759-10769.

[24]Z.-S. Wu, W. Ren, L. Gao, B. Liu, C. Jiang, H.-M. Cheng, Carbon 47 (2009) 493499.

[25]D.-W. Wang, F. Li, M. Liu, G. Q. Lu, H.-M. Cheng, Angewandte Chemie International Edition 47 (2008) 373-376. 
[26]H. Kim, M.-Y. Cho, M.-H. Kim, K.-Y. Park, H. Gwon, Y. Lee, K. C. Roh, K. Kang, Adv Energy Mater 3 (2013) 1500-1506.

[27]G. Wang, L. Zhang, J. Zhang, Chemical Society Reviews 41 (2012) 797-828.

[28] Y. Gogotsi, P. Simon, Science 334 (2011) 917-918.

[29]Y. Shilina, M. D. Levi, V. Dargel, D. Aurbach, S. Zavorine, D. Nucciarone, M. Humeniuk, I. C. Halalay, Journal of the Electrochemical Society 160 (2013) A629A635.

[30]X. Wu, J. Zhou, W. Xing, G. Wang, H. Cui, S. Zhuo, Q. Xue, Z. Yan, S. Z. Qiao, Journal of Materials Chemistry 22 (2012) 23186.

[31]M. D. Stoller, S. Murali, N. Quarles, Y. Zhu, J. R. Potts, X. Zhu, H. W. Ha, R. S. Ruoff, Physical Chemistry Chemical Physics 14 (2012) 3388-3391.

[32]F. Béguin, V. Presser, A. Balducci, E. Frackowiak, Advanced Materials 26 (2014) 2219-2251.

[33]S. Güney, G. Yildiz, A. Capan, T. Ozturk, Electrochimica Acta 55 (2010) 32953300. 